\title{
Cervical dystonia - improving the effectiveness of botulinum toxin therapy
}

\author{
Małgorzata Tyślerowicz ${ }^{1}$, Weronika Kiedrzyńska ${ }^{1}$, \\ Bożena Adamkiewicz ${ }^{1}$, Wolfgang H. Jost ${ }^{2}$, Jarosław Sławek ${ }^{3}$ \\ ${ }^{1}$ Department of Neurology, Copernicus Memorial Hospital in Lodz, \\ Comprehensive Cancer Centre and Traumatology, Lodz, Poland \\ ${ }^{2}$ Parkinson-Klinik Ortenau, Wolfach, Germany \\ ${ }^{3}$ Department of Neurological-Psychiatric Nursing, Medical University of Gdansk, Poland; \\ Department of Neurology, St Adalbert Hospital, Copernicus Ltd, Gdansk, Poland
}

\begin{abstract}
Introduction. Cervical dystonia is the most frequent form of focal dystonia. It is characterised by involuntary muscular contractions resulting in abnormal head/neck and shoulder movements and postures, which can be associated with tremor and pain. Local intramuscular injections of botulinum toxin type $A$ (BoNT-A) is the treatment of choice, being both effective and well-tolerated. However, a considerable number (c. 30\%) of patients discontinue this treatment.

The aim of this review was to analyse the factors possibly responsible for treatment failures of cervical dystonia (CD), with special regard to the new classification known as the 'Col-Cap' concept and non-motor symptoms.

Clinical implications. Several factors analysed in this review are responsible for effective treatment: proper diagnosis of dystonia and exclusion of pseudodystonias, correct recognition of dystonia pattern and identification of new patterns according to the Col-Cap concept, muscle selection and precise injections under electromyography (EMG) and/or ultrasonography (US) guidance. Furthermore, concomitant diagnosis and treatment of non-motor symptoms such as depression, anxiety, fatigue, sleep problems, phobias and stigmatisation are crucial in obtaining the best overall effect of the treatment. Primary and secondary immunisation and non-responsiveness seem to be marginal problems nowadays due to a low potential of new BoNT-A formulations to produce neutralising antibodies.

Future directions. There is a need for new and relevant scales combining the Col-Cap concept patterns with non-motor symptoms and quality of life. There is also a lack of specific rehabilitation protocols which could enhance BoNT-A treatment results.
\end{abstract}

Key words: cervical dystonia, Col-Cap concept, botulinum toxin, treatment failures, primary non-responsiveness, secondary non-responsiveness

(Neurol Neurochir Pol 2020; 54 (3): 232-242)

\section{Introduction}

Cervical dystonia $(\mathrm{CD})$ is a movement disorder characterised by involuntary, sustained or intermittent muscle contractions leading to abnormal head movements and/or positioning. It is accompanied by pain in $67-75 \%$ of patients [1]. Typical features include sensory trick and head tremors. A substantial number of patients (18-41\%) demonstrate complications manifesting in premature degenerative disorder of the cervical spine, discopathy or cervical myelopathy [2]. CD significantly reduces quality of life, affects the ability to work, and socially stigmatises patients [2-3]. Spontaneous remission occurs in up to $15 \%$ of patients and is usually temporal $[5,6]$, but may be triggered by botulinum neurotoxin type A (BoNT-A) treatment [4]. CD is the most common focal dystonia: a meta-analysis reveals that it affects a mean 4.98 of every 100,000 people. However, a significant difference has been observed with regards to geographical location: in Japan the rate is 2.52, whereas in Europe it is 6.71 [5]. 
Table 1. Summary of possible causes of non-responsiveness or unsatisfactory effect of Botulinum toxin type A treatment of cervical dystonia

1. Primary non-responsiveness

- preexisting BoNT-A antibodies, chronic exposure to BoNT-A in childhood, cross-reaction of other antibodies (e.g. tetanus toxin)

\section{Secondary non-responsiveness}

- neutralizing antibodies

- change in the pattern of dystonia, conditioned by the central mechanism of non-specific muscle activation

\section{Misdiagnosis}

- dystonia in neurodegenerative diseases (PSP — usually retrocollis, MSA — usually anterocollis)

- genetic disorders: Wilson's disease, Huntington's disease, spinocerebellar ataxias, neuroacanthocytosis, NBIA

- dystonia in mitochondrial disorders

- secondary dystonia caused by identified factors: post-traumatic dystonia, post-stroke dystonia, post-inflammatory dystonia, tardive dystonia, toxin-related dystonia

- functional dystonia (psychogenic)

- pseudodystonias:

a. related to musculoskeletal deformations: camptocormia, scoliosis, Grisel's syndrome, Arnold-Chiari syndrome, Klippel-Feil syndrome, joints deformities, arthrogryposis, Dupuytren's contracture, congenital muscular torticollis, Sandifer's syndrome,

b. related to the compensation of improper functioning of the central nervous system or peripheral nervous system (compensatory head tilt): vestibular system disorder, oculomotor nerves palsies $\left(6^{\text {th }}, 4^{\text {th }}\right)$, mass lesion in the posterior fossa

c. disorders of sensory pathways: parietal lobe damage, syringomyelia, myelopathy, mono- and polyneuropathy

d. disorders of motor pathways: Isaac's syndrome, stiff-person syndrome, tetanus, myotonic disorders, MMN

4. Misidentification of the subtype of dystonia (new classification-according Col-Cap concept and new CD patterns with involvement of muscles not routinely injected earlier)

5. Improper selection of active muscles (injections of muscles that are not responsible for specific pattern) and missing the muscles (no guidance, too short needles, too deep injections missing thin muscle layers, e.g. m. trapezius)

6. Lack of monitoring techniques of injections: us, emg (combination is the optimal method as visualization does not mean that muscle is really active)

7. Improper adjustment of the total dose and its distribution in particular muscles

8. Subjective feeling of lack of improvement (dominating non motor symptoms like depression, anxiety, phobias or sleep problems)

9. Long-lasting dystonias causing secondary changes like myofibrosis, contractures

10. Improper storage and transportation of the medication

PSP — Progressive Supranuclear Palsy, MSA — Multiple System Atrophy, NBIA — Neurodegeneration with Brain Iron Accumulation, MMN - Multifocal Motor Neuropathy

Treatment of CD with BoNT-A is the treatment of choice as recommended by the American Academy of Neurology (AAN), with high effectiveness and safety profiles [6].

Double-blind, randomised clinical, as well as open, studies have shown that $50-85 \%$ of patients demonstrate a significant improvement [7-15]. A considerable number (c. 30\%, range 19-46\%) of patients discontinue treatment once it appears to be ineffective [16-20]. Treatment may be considered ineffective if neither the patient nor the therapist have observed a satisfactory reduction of symptoms or if significant adverse effects occur (e.g. dysphagia or neck muscles weakness), or when clinical trials observe no significant score reduction in rating scales. Those most commonly used are the Tsui Scale and the Toronto Western Spasmodic Torticollis Rating Scale, TWSTRS.

This article aims to analyse the possible factors responsible for treatment failures, with special regard to the new classification of CD known as the Col-Cap concept and non-motor symptoms.

\section{Making a proper diagnosis}

BoNT-A can be effective both in primary and secondary dystonia. However, the latter may require specific treatment to avoid fatal progression and outcome like e.g. Wilson's disease.
Therefore, a proper diagnosis is essential. Particular attention should be paid to pseudodystonias that mimic dystonia and do not respond to treatment. Revised definitions and an extended list of pseudodystonic postures was recently presented by Berlot et al. (Tab. 1) [21]. Pseudodystonic postures must be clearly differentiated from conditions related to muscles weakness which can result in a head-drop e.g. myasthenia, muscular dystrophy, and amyotrophic lateral sclerosis. Moreover, pathological anteflexion can be confusing (e.g. anteflexion in multiple system atrophy) because there is no consensus as to whether it is related to dystonia of flexors or myositis and weakness of neck extensors [22]. It has been speculated that neck extensor myopathy might follow mechanical over-stretching from flexor dystonia. There is evidence that even muscles which are not under mechanical stress can develop myopathic changes. Pathologic changes seen in inflammatory myopathies (IBM) have been found in clinically affected paraspinal muscles. This shows the neurodegenerative nature of both IBM and parkinsonism.

Additionally, functional CD may be refractory to BoNT-A treatment. It usually presents as fixed dystonia or is multidirectional, variable with different patterns at the same time, accompanied by enormous effort put into head positioning, with improvement after distraction, and no sensory twitches. 


\section{Proper identification of dystonia pattern}

One of the most common reasons behind therapy failures seems to be incorrect identification of the clinical pattern of $\mathrm{CD}$. To properly identify the subtype of dystonia, the patient should be examined carefully, not only in a relaxed seated position when their upper body is in a resting state, but also with open and closed eyes (because closing eyes can worsen dystonic posture), standing and/or lying positions, while walking or performing activities such as writing (these can enhance dystonic movements).

By observing the patient from the side, the front, and also from the back, we can assess the change in head and neck position in each of the three planes. Sometimes an objective assessment may be difficult due to the activity of compensating muscles as well as coexistence of tremors and/or myoclonus. We should pay attention to the so-called 'sensory twitches' (as they may change the pattern temporarily), shoulder and scapula positions, and potential muscle hypertrophy and pain. If the arm is elevated, we should assess the patient by stabilising their arms by pushing down the elevated arm. This may reveal its compensating character to maintain the head erect position due to a severe head tilt.

The Col-Cap concept was first set out by Reichel et al. in 2009 [23]. Their careful examination identified new patterns not previously recognised. Initially, $\mathrm{CD}$ had been classified into four types. These related to: turning the head (torticollis), tilting the head to one side (laterocollis), backwards (retrocollis), or forwards (anterocollis). More than 50\% of cases were diagnosed as torticollis, $10-15 \%$ as laterocollis or retrocollis, and less frequently anterocollis [24]. The 'Col-Cap' concept (collum-caput $=$ neck-head $)$ was invented on the basis of imaging examinations (CT/MRI of the head, cervical spine and also soft tissues) and functional anatomy. According to this approach, based on the various movements of the head (muscles insertions between skull and C2 spine level) and neck (muscles insertions between C2 and C7 level), 10 major subtypes of $\mathrm{CD}$ were identified (Fig. 1): transverse (torticaput/ /collis), frontal (laterocaput/collis), sagittal with tilting forwards (anterocaput/collis) or backwards (retrocaput/collis) $[25,26]$. These distinctive patterns are 'realised' by the activity of different (i.e. different from those in the 'classic four') muscles involved in the particular type. So an inaccurate diagnosis may result in a lack of effect. According to the multicentre study recently published by Jost et al. analysing 306 consecutive patients with $C D$, pure forms are rare $(16.3 \%)$, whereas combinations of 2-6 of the subtypes are common (83.7\%). Among all the subtypes, the most common primary form is torticaput (49\%), and the second most common is laterocaput (16.7\%) [27]. One can also distinguish combinations of incorrect positions such as a mixture of laterocollis to one side and laterocaput in the opposite direction; this is known as lateral shift. In addition, we see a combination of anterocollis and retrocaput called anterior sagittal shift, and consequently posterior sagittal shift as a combination of retrocollis and anterocaput.

To identify these new patterns, characteristic points/ /lines should be identified, which enables levels of movement (collis, caput) to be distinguished. In the case of torsion, the main anatomical structures are: superior thyroid incisure of the larynx and manubrium of the sternum above the jugular incisure. Rotational torticollis is diagnosed if, during rotation, the larynx shifts in relation to the sternum. If these points remain in the same line, but the chin shifts in relation to the larynx, rotational head position is diagnosed (torticaput). In the sagittal plane, the meatus acusticus externus and the clavicula are useful anatomical landmarks. If only the 'head' is concerned, the meatus acusticus stays in line with the clavicula (anterocaput, retrocaput). If the projection is in front or behind the clavicula, the 'neck' level is involved (anerocollis/anterocaput) (Fig. 1) [28]. A treatment protocol differentiating the head and the neck level may result in better outcomes. However, to date this has only been shown in one retrospective study [29].

Based on the Col-Cap concept, 'main' muscles should be injected as the first choice, then 'secondary' in each subtype. In complex patterns, the leading (or primary) one should be first injected and in refractory cases previous injection patterns should be assessed and modified in the subsequent cycle [29].

\section{Correct selection of active muscles and guided injections (US/EMG)}

Before the Col-Cap concept, muscles were selected on the basis of functional anatomy, a physical examination accompanied by assessment of muscle hypertrophy, location of pain, or arm elevation. Several published studies used electromyography (EMG) as an injection technique for the identification and localisation of muscles [30-34]. There are studies which strongly support the role of EMG showing that injections performed only according to anatomical landmarks can be imprecise $(83 \%$ reached the sternocleidomastoid, but only $47 \%$ the levator scapule muscle) [35]. Moreover, EMG guidance increases the sensitivity and specificity of the muscle selection, even when performed by BoNT-A specialists. Clinical predictions of individual muscle involvement are only 59\% sensitive and 75\% specific without EMG use. It has been pointed out that muscular hypertrophy or shoulder elevation indicates 'dystonic' muscle activity in only $70 \%$ of patients, showing how a classical physical examination can be confusing [36].

The role of EMG has been supported by randomised, blinded studies which have demonstrated that EMG-guided injections vs. anatomically-based bring significantly better results, measured by rating scales and subjective assessment: $82 \%$ (TWSTRS) and 61\% (patients assessment) vs. 8\% (TWSTRS) and 25\% (patient report) respectively [34]. The same was proved in another study, which showed a significant difference in Jankovic scale $(\mathrm{p}=0.05)$ between guided and blinded injections [37]. Retrospective analysis confirmed the benefits of 


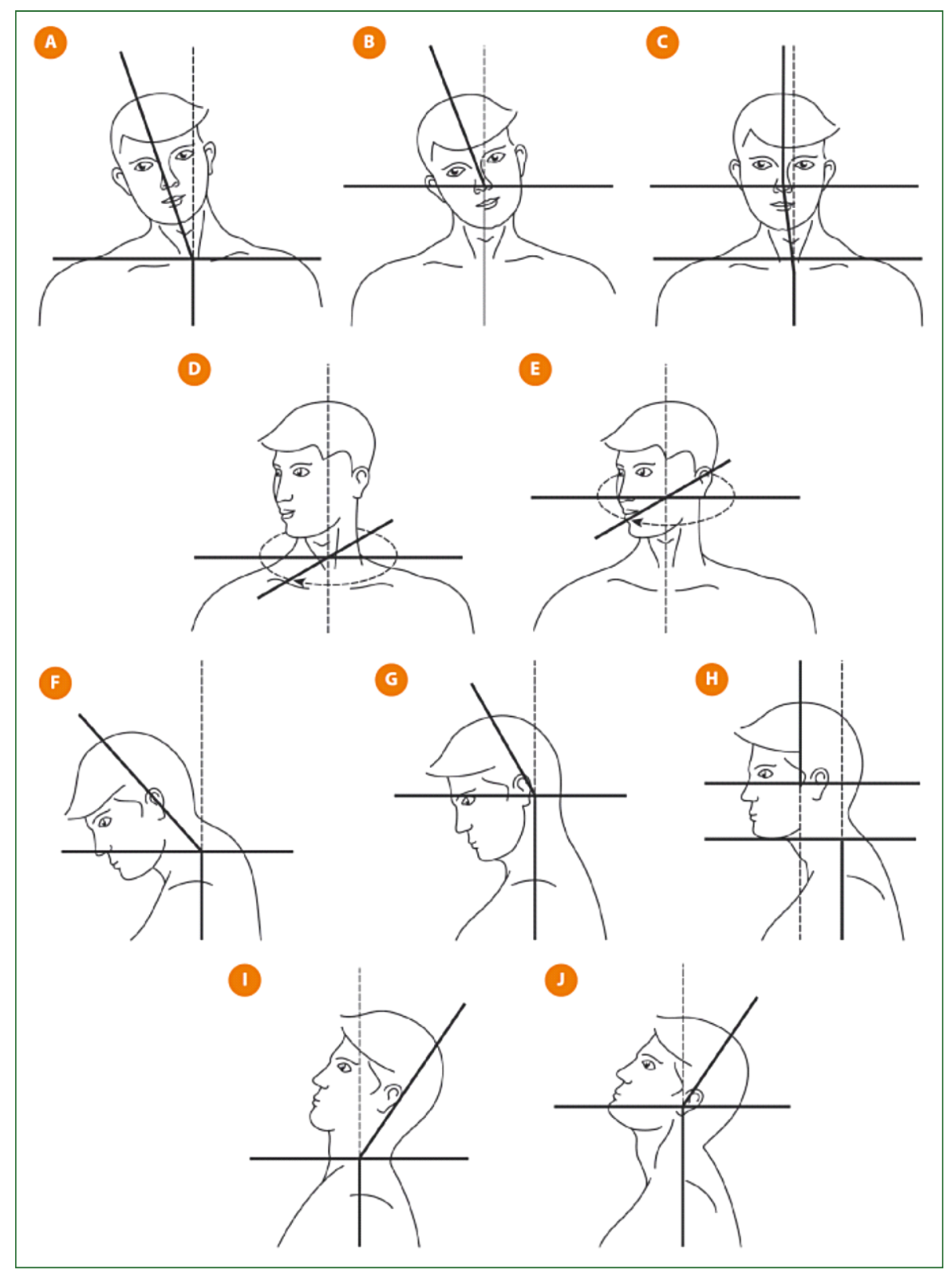

Figure 1. Ten basic clinical patterns of cervical dystonia according to col-cap concept. A. Laterocollis, B. Laterocaput, C. Lateral shift (combination of laterocillis to one and laterocaput to opposite side), D. Torticollis, E. Torticaput, F. Anterocollis, G. Anterocaput, H. Sagittal-anterior shift (combination of anterocollis and retrocaput), I. Retrocollis, J. Retrocaput. Additional rare variant may be present (no picture) presenting as Sagittal posterior shift (combination of retrocollis and anterocaput). Courtesy of Via Medica and Jarosław Sławek, Monika Rudzińska eds, In: Toksyna botulinowa w praktyce neurologicznej, Via Medica, Gdańsk 2015, vol. 1, Fig. 2

using polymyography in groups of patients previously treated with BoNT with an unsatisfactory response. After one year of treatment, reasonable or good final results were obtained in $60 \%$ of patients measured on both the Tsui Scale $(\mathrm{p}<0.01)$ and the subjective assessment of patients $(\mathrm{p}<0.001)$ [38]. Subsequently, one small open trial showed that EMG improved the treatment outcome in $9 / 10$ patients, demonstrating a significant improvement in TWSTRS (mean improvement of 64\%) [39]. In contrast, there are studies which do not confirm the utility of EMG guided injections. In a systematic review by Nijmeijer et al., the average improvement on the Tsui Scale was greater (31.9 vs. $43.7 \%$ ) in studies that used only clinical evaluation [40]. But because of profound differences in methodology, dosage, patient characteristics, and primary and secondary endpoints, no statistical analysis could be performed, and no firm conclusions could be drawn.

To sum up, a consensus of experts recommends a combination of clinical assessment and EMG examination as well 
as EMG-guided injections in patients treated for the first time (Level A) $[34,41]$. But for those who have undergone unsuccessful treatment, EMG usefulness is less proven (Class III studies and Level C) [42, 43].

We must remember that EMG use does not allow us to distinguish between 'dystonic' and compensatory muscle activities or to visualise the tip of the needle (the injector is not sure if the needle is positioned in the intended muscle). Such precise targeting of muscles can be achieved using other methods such as ultrasonography (US) or computed tomography (CT).

Ultrasound is an easily available, non-invasive method enabling the visualisation of muscles and surrounding structures (nerve bundles and large vessels) in real time, which may increase the accuracy of injections of not only deep, but also superficial (sometimes as superficial as for example very flat trapezius) muscles. Studies regarding spasticity treatment with BoNT under US guidance have shown its efficacy in localising especially deep muscles. Recent publications are open studies conducted in small groups of patients or case studies on injections of deeply located muscles where the approach is challenging, such as the longus colli muscle or obliquus capitis inferior [41-44]. One of these studies assessed the impact of monitoring techniques such as US and/or CT in a group of eight patients requiring injections in deep cervical muscles (the obliquus capitis inferior, the longus colli muscle, obliquus capitis superior, scalenus anterior and scalenus posterior). The Tsui Scale confirmed a significant improvement occurring within four weeks (11.75 vs. 1.50) and on the TWSTRS scale in each of the subscales ( 20.0 vs. $5.25,20.0$ vs. 7.00 , and 13.10 vs. 6.50 ) [48]. A study conducted in a group of five patients to assess the incidence of swallowing problems after injections revealed that an ultrasound examination, carried out in order to locate the EMG needle during injections in the sternocleidomastoid, significantly reduced such adverse effects ( $0 \%$ vs. $34.7 \%$ ) [45]. Nevertheless, no randomised, controlled studies have proved the greater effectiveness of US-guided versus blinded injections.

Muscles which seem to 'benefit' mostly from US monitoring include: suprahyoid muscles, scalenus muscles, the longissimus capitis and cervicis, semispinalis capitis and cervicis, obliquus capitis inferior (crucial muscles in some col-cap patterns), but also the sternocleidomastoid, levator scapulae, and trapezius (frequently injected too deeply) [46].

It seems that in some cases, particularly with accompanying tremors or after several non-effective treatment attempts, EMG and US methods, applied simultaneously, should be considered [47]. The needle size should also be adjusted because one that is too short will not reach deep muscle layers, although these can be easily detected thanks to US guidance.

\section{Optimal dosage and its distribution in particular muscles}

The optimal dose of BoNT-A was obtained in pivotal studies. The recommendations according to SPC (summary product characteristics) are based on these clinical trials. However, dosage should be adjusted in subsequent treatment cycles both in terms of effectiveness and safety. There are studies which indicate that efficacy and the incidence of side effects depend on the BoNT-A dose. A study assessing abobotulinumtoxin A showed that the largest and longest improvements were obtained in the $1,000 \mathrm{U}$ group [12]. Nevertheless, the highest number of adverse events also occurred in this group. All groups (placebo, 250U, 500U and 1,000U) demonstrated improvements $>20 \%$ on the Tsui Scale after two weeks. However, in week 4 such improvements were still observed only in the $500 \mathrm{U}$ and the 1,000 U groups. In these groups, also compared to the $250 \mathrm{U}$ and placebo groups, patients reported $>50 \%$ improvement on CGI scale statistically more frequently. Therefore, the experts' recommendations, based on those studies $[12,48]$, suggest starting treatment by administering $500 \mathrm{U}$ of abobotulinumtoxin. However, lower (200-400 U) doses might be equally effective and safe if precisely administered e.g. under EMG guidance [49]. In clinical practice, the dose should be adjusted to muscle bulk and body mass, although formal studies have not been performed.

There are no randomised, controlled studies comparing the effectiveness of the number of injections per muscle. A comparative study on 49 patients showed that multi-point injections increased treatment effectiveness: they reduced pain $(\mathrm{p}<0.002)$, increased the range of motion $(\mathrm{p}<0.001)$, and lengthened the duration of effect $(\mathrm{p}<0.001)$ [50]. Experts recommend distributing the dose to 1-4 points, depending on the area of the muscle [51].

\section{Subjective feelings of lack of improvement and non-motor symptoms}

Patients suffering from cervical dystonia, like those with other movement disorders, present a wide spectrum of non-motor symptoms which have not been considered in clinical trials assessing BoNT-A effectiveness, but may influence the overall result of therapy.

Patients with $\mathrm{CD}$ appear to be more aware of having abnormal dyskinetic movements than do patients with Huntington's or Parkinson's Diseases [52]. Non-motor symptoms have been noted in several studies: $61.8 \%$ of patients with CD presented lack of self-confidence due to stigmatisation, $59.8 \%$ had sleep problems, and 51\% fatigue [53]. Depression was prevalent in as many as $47.5 \%$ of patients and this was the major determinant of poor quality of life [3]. In another study, poor quality of life was more common in CD than in blepharospasm and writer's cramp and also depression and anxiety were the major correlates [54].

Depression, along with other emotional-psychological disorders, may constitute a clinical spectrum of CD independently of motor symptoms. Mood disturbances coupled with anxiety, also adjustment disorders or obsessive-compulsive behaviours, occur significantly more often in patients with focal dystonias 
(57.3\% of patients with dystonia, compared to $24.1 \%$ of healthy subjects and $34.6 \%$ of patients with hemifacial spasm) [55]. Another study showed that patients with $\mathrm{CD}$ are much more frequently affected by depression (15-53.4\%) and anxiety disorders (26.4-83.3\%), and 4.5 times more often by agoraphobia or panic attacks than the general population. No correlation was found between age, duration of dystonia or its severity, as well as duration of BoNT-A treatment, which may indicate that mental disorders are primary, but not secondary, to dystonia.

A study by Berardelli et al. showed that during a five-year follow up of treatment with BoNT injections, it significantly improved dystonic movements (TWSTRS $33.4 \pm 11.1$ at baseline, $26.9 \pm 10.9$ after five years). However, the incidence of neuropsychiatric disorders did not improve at all (65\% at baseline, $64 \%$ after five years), which suggests an independent mechanism and, possibly, the need for additional treatment [56]. A similar effect was observed in Sławek et al's study, showing in a group of 101 patients with CD treated with BoNT-A that size effect for TWSTRS (motor presentation) was significant after treatment: 1.1 ( $\mathrm{SD} \pm 0.6$ ), but for depression (Montgomery Åsberg Depression Rating Scale) it was only $0.5(\mathrm{SD} \pm 0.7)[4]$.

Stamelou et al. presented evidence indicating an important non-motor component to primary dystonia, including abnormalities in sensory and perceptual functions, as well as the neuropsychiatric, cognitive and sleep domains [57]. Widespread loss of inhibition and pathologically increased plasticity appear to play important roles in the pathophysiology of primary dystonia [58]. The hypothesis is that non-motor features of dystonia could be explained by a common pathophysiological deficit that also underlies the motor symptoms [59-61]. Genetic susceptibility is the key to the pathophysiology of dystonia, indicated by the numerous non-motor abnormalities that are found in unaffected first-degree relatives of patients with adult-onset focal dystonia and non-manifesting gene mutations carriers. This genetic background may predispose patients to develop dystonia in the presence of other factors that may have important non-motor components, such as repetitive activity, trauma, or emotional distress [61].

Considering the accompanying non-motor disorders, such as pain or a broad spectrum of psychiatric diseases, it seems that analgesics or antidepressants would be beneficial. There are, however no controlled studies supporting such practice. The only randomised, controlled trial conducted recently looked at the efficacy of escitalopram in the treatment of $\mathrm{CD}$ with concomitant tremor. It did not reveal any beneficial effects of the drug on either motor or non-motor symptoms; the authors underline however that this should not be a reason for resigning from such therapy [62].

\section{Primary and secondary immunoresistance}

Patients who do not respond to the therapy can be classified as those who did not respond at all from the beginning (so-called primary non-responders, PNR), or those who stopped responding to the treatment after a good initial effect (so-called secondary non-responders, SNR).

It is suspected that primary resistance may be associated with preexisting BoNT-A antibodies $(\mathrm{AB})$, chronic exposure to BoNT-A in childhood, or cross-reaction of other $A B$ (tetanus toxin $A B)[63,64]$. However, this is only speculation unsupported by studies. Secondary non-responsiveness is defined differently by different authors. Some authors claim that two consecutive ineffective treatment cycles (i.e. no subjective improvement or exacerbation by at least 2 points on the Tsui Scale as well as absence of side effects typical for BoNT-A) which occur after at least two effective cycles in the past (defined as improvement on the Tsui Scale by at least 3 points and/ /or atrophy in injected muscles and/or an occurrence of side effects typical for BoNT-A) are enough to diagnose secondary resistance [18]. Others believe that secondary resistance can be diagnosed only after three consecutive ineffective treatment cycles [65]. One should remember that it may be a pseudoimmunoresistance due to other reasons mentioned earlier, and that the real one should be confirmed in laboratory or clinical tests (see below).

It is estimated that secondary resistance affects approximately $3-5 \%$ of patients [66]. Previously the production of $A B$ to BoNT-A was considered the main cause of secondary treatment failure (STF). Some publications indicate that higher BoNT-A doses, administered at shorter intervals and frequent injections (within six weeks of the previous injection, so called booster injections), increase this risk of development of $\mathrm{AB}[67,68]$. Most of the previous studies reporting $A B$ in groups of $C D$ patients were unbiased regarding STF and had a short duration. Therefore, $\mathrm{AB}$ rates reported in these studies more or less represent the incidence of $A B$ induction during 1-2 years of therapy and the range is between $0.5 \%$ and $2.0 \%$ [69-71]. In long-term treated patients with $\mathrm{CD}$, the prevalence of $\mathrm{AB}$ is higher, indicating $14 \%$ in the group of patients still responding to therapy who underwent BoNT-A injections over 10 years [72]. Another study showed that duration of treatment of $\sim 15$ years carries a risk of up to $40 \%$ of becoming $\mathrm{AB}$ positive, not influencing the treatment result. In addition, the study showed that single dose per session and BoNT-A formulation were the most significant factors influencing $\mathrm{AB}$ formation [73].

Furthermore, the amount of complexing proteins, which differs in the three most commonly used preparations, plays an essential role. Antibodies were found in $9.5 \%$ of patients treated only with original onabotulinumtoxinA with a large amount of complexing proteins ( $100 \mathrm{U} / 25 \mathrm{ng}$ protein), whereas $\mathrm{AB}$ were not detected in any patients treated exclusively with the new preparation of onabotulinumtoxinA (100U/5 ng protein) marketed more than 20 years ago [74]. Antibodies were not found in patients treated with incobotulinumtoxinA, which does not contain complexing proteins $[75,76]$.

Neutralising antibody titre decreases after discontinuation of therapy. The rate of decline differs for each individual, and 
the decline can last for up to four years. Minimum 12-week intervals between injections are still advisable. However, some studies on incobotulinumtoxinA reveal that shorter intervals are also safe and do not produce antibodies [19].

In summary, studies show that $\mathrm{AB}$ and their titre do not necessarily contribute to secondary resistance; subsequent studies revealed that antibodies are found in approximately $50 \%$ of patients with secondary resistance $[77,78]$ and the reasons for this situation possibly differ.

According to some studies, secondary resistance could be associated with a higher dose of BoNT-A, administration of other therapies (rehabilitation, pharmacological treatment), a significant number of side effects, and more frequent interruptions in BoNT-A treatment [79].

The lack of response due to the neutralising antibodies formation may be diagnosed with specific tests. MPA (mouse protection assay), HDA (hemidiaphragm assay (HDA), and new enzyme-linked immunosorbent assay (ELISA) are currently available. According to the latest reports, a combination of ELISA and HDA is a quick method characterised by the highest sensitivity and its price is reasonable. For $100 \%$ sensitivity its specificity is $90 \%$, and for $100 \%$ specificity its sensitivity is $55 \%$. Sensitivity of previously used tests, such as MPA or WBA (western blot assay) was lower and ranged from $33 \%$ to $53 \%[80]$.

Instead of laboratory tests, in clinical practice we can use simple, objective tests involving a unilateral application of BoNT-A in the frontal muscle [74] or in the extensor digitorum brevis muscle. The frontal test is performed by administering 30 units of abobotulinumtoxinA or 10 units of ona/incobotulinumtoxinA [71] in the frontalis muscle on one side. Clinical assessment (ability to raise eyebrows) is recommended after 2-4 weeks. Asymmetry indicates that BoNT-A is effective [43]. For an extensor digitorum brevis muscle test we administer $100 \mathrm{U}$ of abo- or 20 units of onabotulinumtoxinA in this muscle and assess the compound muscle action potential response (CMAP) by stimulating the peroneal nerve at baseline and two weeks after injection (CMAP should more than halve compared to its original value). For a decline of up to $20 \%$, immunoresistance is quite probable; for values of $20-50 \%$, the result is doubtful [81].

In patients with immunoresistance, we can discontinue injections for 12-18 months and observe the patient at regular intervals. If dystonia significantly reduces quality of life, an alternative is re-administration of BoNT-A. Or one may consider the use of botulinum toxin $B$, which is safe and effective in the treatment of cervical dystonia, but presents high immunogenicity potential, in particular in patients already resistant to BoNT-A treatment $[82,83]$.

\section{Intrinsic muscle changes and change of dystonia pattern}

Muscle fibrosis and contractures, being the result of long-lasting disease as well as a change in the pattern of dystonia, play an important role in secondary resistance apart from immunisation. The muscular dystonia pattern may change in some patients over time. BoNT-A injections may 'activate' other previously inactive muscles (contributing to the similar clinical pattern of dystonia), which implies the activity of a central mechanism, conditioning the position of the head or neck through non-specific muscle activation. The clinical pattern of dystonia in the course of the disease can also change. This probably results from activation of other muscles, which in turn results from a peripheral block of initially active dystonic muscles, or a change of the activation centre at the level of the central nervous system [84-86].

\section{Evaluating the effectiveness of treatment}

A lack of improvement in a patient's assessment may contradict the positive change in rating scales. The most commonly used are Tsui, CIDP-58 and TWSTRS.

The TWSTRS is most commonly used in clinical trials and serves as the primary endpoint for assessing the effectiveness of BoNT-A in treating CD [14, 17, 87-90]. In most studies, an improvement by $25-30 \%$ measured with this scale is considered significant. It shows a strong correlation with the Tsui Scale [91]. Despite many advantages however, it does not take into account the evaluation of dystonic tremor; there is no clear definition of the midline and the full range of motion [92]. This scale also cannot assess properly the complex patterns of CD demonstrated in the Col-Cap concept.

In addition, due to its complex nature, the scale can hardly ever be applied in everyday practice. The authors of one recent study determined the number of points in the TWSTRS scale which contributes to a minimally clinically perceptible change in the Patient Global Impression of Change (PGIC) scale: a change by 3 points in milder cases $(\leq 28.5)$ and 18 points in severe cases $(>52)$ [93]. A linear relationship was also found between the TWSTRS total scores and the PGIC: an improvement by 2.9 points in the TWSTRS scale corresponded to a change by one category in the PGIC scale.

The lack of a specified rating scale including both motor (with respect to the new Col-Cap patterns) and wide spectrum of non-motor CD features seems to be an unmet need. Moreover, it may create difficulties when planning new studies [94].

The next unmet need is the lack of consensus on specific rehabilitation programmes dedicated to $\mathrm{CD}$ patients and aimed at enhancing the effect of BoNT-A therapy.

In conclusion, $\mathrm{CD}$ treatment with BoNT-A remains a challenge for the physician. It is rare to obtain satisfactory effects at the first session. If unsuccessful, the long list of possible reasons, which have been the subject of this paper, should be considered.

Conflicts of interest: All authors declare no conflict of interest

Funding: No funding sources 
Ethical committee permission: Not necessary as this paper is a review, with no participation by patients or animals and no experiments

\section{Acknowledgements: None}

\section{References:}

1. Kutvonen 0 , Dastidar P, Nurmikko T. Pain in spasmodic torticollis. Pain. 1997; 69(3): 279-286, doi: 10.1016/s0304-3959(96)032964, indexed in Pubmed: 9085302.

2. Konrad C, Vollmer-Haase J, Anneken $\mathrm{K}$, et al. Orthopedic and neurological complications of cervical dystonia-review of the literature. Acta Neurol Scand. 2004; 109(6): 369-373, doi: 10.1111/j.1600-0404.2004.00281.x, indexed in Pubmed: 15147457.

3. Sławek J, Friedman A, et al. Factors affecting the health-related quality of life of patients with cervical dystonia and the impact of botulinum toxin type A injections. Funct Neurol 2007 22(2):95-100.

4. Jankovic J, Leder S, Warner D, et al. Cervical dystonia: clinical findings and associated movement disorders. Neurology. 1991; 41(7): 10881091, doi: 10.1212/wnl.41.7.1088, indexed in Pubmed: 2067638.

5. Friedman A, Fahn S. Spontaneous remissions in spasmodic torticollis. Neurology. 1986; 36(3): 398-400, doi: 10.1212/wnl.36.3.398, indexed in Pubmed: 3951708.

6. Mainka T, Erro R, Rothwell J, et al. Remission in dystonia - Systematic review of the literature and meta-analysis. Parkinsonism Relat Disord. 2019; 66: 9-15, doi: 10.1016/j.parkreldis.2019.02.020, indexed in Pubmed: 30898428.

7. Giladi $\mathrm{N}$, Meer J, Kidan H, et al. Long-term remission of idiopathic cervical dystonia after treatment with botulinum toxin. Eur Neurol. 2000; 44(3): 144-146, doi: 10.1159/000008223, indexed in Pubmed: 11053961.

8. Steeves TD, Day L, Dykeman J, et al. The prevalence of primary dystonia: a systematic review and meta-analysis. Mov Disord. 2012; 27(14): 1789-1796, doi: 10.1002/mds.25244, indexed in Pubmed: 23114997.

9. Simpson DM, Hallett M, Ashman EJ, et al. Practice guideline update summary: Botulinum neurotoxin for the treatment of blepharospasm, cervical dystonia, adult spasticity, and headache: Report of the Guideline Development Subcommittee of the American Academy of Neurology. Neurology. 2016; 86(19): 1818-1826, doi: 10.1212/ WNL.0000000000002560, indexed in Pubmed: 27164716.

10. Greene P, Kang U, Fahn S, et al. Double-blind, placebo-controlled trial of botulinum toxin injections for the treatment of spasmodic torticollis. Neurology. 1990; 40(8): 1213-1218, doi: 10.1212/wnl.40.8.1213, indexed in Pubmed: 2199847.

11. Poewe W, Deuschl G, Nebe A, et al. What is the optimal dose of botulinum toxin $A$ in the treatment of cervical dystonia? Results of a double blind, placebo controlled, dose ranging study using Dysport. German Dystonia Study Group. J Neurol Neurosurg Psychiatry. 1998; 64(1): 13-17, doi: 10.1136/jnnp.64.1.13, indexed in Pubmed: 9436721.

12. Brans JW, Lindeboom R, Snoek JW, et al. Botulinum toxin versus trihexyphenidyl in cervical dystonia: a prospective, randomized, double-blind controlled trial. Neurology. 1996; 46(4): 1066-1072, doi: 10.1212/wnl.46.4.1066, indexed in Pubmed: 8780093.

13. Truong D, Duane DD, Jankovic J, et al. Efficacy and safety of botulinum type A toxin (Dysport) in cervical dystonia: results of the first US randomized, double-blind, placebo-controlled study. Mov Disord. 2005;
20(7): 783-791, doi: 10.1002/mds.20403, indexed in Pubmed: 15736159.

14. Comella C, Jankovic J, Truong D, et al. Efficacy and safety of incobotulinumtoxinA (NT 201, XEOMIN®, botulinum neurotoxin type A, without accessory proteins) in patients with cervical dystonia. Journal of the Neurological Sciences. 2011; 308(1-2): 103-109, doi: 10.1016/j. jns.2011.05.041.

15. Evidente VG, Pappert EJ. Botulinum toxin therapy for cervical dystonia: the science of dosing. Tremor Other Hyperkinet Mov (N Y). 2014; 4: 273, doi: 10.7916/D84X56BF, indexed in Pubmed: 25414799.

16. Truong D, Brodsky M, Lew M, et al. Global Dysport Cervical Dystonia Study Group. Long-term efficacy and safety of botulinum toxin type A (Dysport) in cervical dystonia. Parkinsonism Relat Disord. 2010; 16(5): 316-323, doi: 10.1016/j.parkreldis.2010.03.002, indexed in Pubmed: 20359934.

17. Charles D, Brashear A, Hauser RA, et al. CD 140 Study Group. Efficacy, tolerability, and immunogenicity of onabotulinumtoxina in a randomized, double-blind, placebo-controlled trial for cervical dystonia. Clin Neuropharmacol. 2012; 35(5): 208-214, doi: 10.1097/ WNF.0b013e31826538c7, indexed in Pubmed: 22948497.

18. Evidente VG, Fernandez HH, LeDoux MS, et al. A randomized, double-blind study of repeated incobotulinumtoxin $A(X e o m i n(囚))$ in cervical dystonia. J Neural Transm (Vienna). 2013; 120(12): 1699-1707, doi: 10.1007/s00702-013-1048-3, indexed in Pubmed: 23779062.

19. Kessler KR, Skutta M, Benecke R. Long-term treatment of cervical dystonia with botulinum toxin A: efficacy, safety, and antibody frequency. German Dystonia Study Group. J Neurol. 1999; 246(4): 265-274, doi: 10.1007/s004150050345, indexed in Pubmed: 10367694.

20. Brashear A, Bergan K, Wojcieszek J, et al. Patients' perception of stopping or continuing treatment of cervical dystonia with botulinum toxin type A. Movement Disorders. 2001; 15(1): 150-153, doi: 10.1002/1531-8257(200001)15:1<150::aid-mds1024>3.0.co;2-x.

21. Hsiung GYR, Das SK, Ranawaya R, et al. Long-term efficacy of botulinum toxin $A$ in treatment of various movement disorders over a 10year period. Mov Disord. 2002; 17(6): 1288-1293, doi: 10.1002/ mds.10252, indexed in Pubmed: 12465070.

22. Skogseid IM, Kerty E. The course of cervical dystonia and patient satisfaction with long-term botulinum toxin A treatment. Eur J Neurol. 2005; 12(3): 163-170, doi: 10.1111/j.1468-1331.2004.01053.x, indexed in Pubmed: 15693803.

23. Gill CE, Manus ND, Pelster MW, et al. Continuation of long-term care for cervical dystonia at an academic movement disorders clinic. Toxins (Basel). 2013; 5(4): 776-783, doi: 10.3390/toxins5040776, indexed in Pubmed: 23612751.

24. Berlot R, Bhatia KP, Kojović M. Pseudodystonia: A new perspective on an old phenomenon. Parkinsonism Relat Disord. 2019; 62: 44-50, doi: 10.1016/j.parkreldis.2019.02.008, indexed in Pubmed: 30819557.

25. Finsterer J, Maeztu C, Revuelta GJ, et al. Collum-caput (COL-CAP) concept for conceptual anterocollis, anterocaput, and forward sagittal shift. J Neurol Sci. 2015; 355(1-2): 37-43, doi: 10.1016/j. jns.2015.06.015, indexed in Pubmed: 26088286.

26. Reichel G, Stenner A, Jahn A. Zur Phänomenologie der zervikalen Dystonien. Fortschritte der Neurologie - Psychiatrie. 2009; 77(05): 272-277, doi: 10.1055/s-0028-1109416.

27. Benecke R, Moore P, Dressler D, et al. Cervical and Arial dystonia. Handbook of botulinum toxin treatment. Blackwell Science, Massachusetts. ; 2003: 158-194. 
28. Reichel G. Cervical dystonia: A new phenomenological classification for botulinum toxin therapy. Basal Ganglia. 2011; 1(1): 5-12, doi: 10.1016/j.baga.2011.01.001.

29. Jost WH, Tatu L. Selection of Muscles for Botulinum Toxin Injections in Cervical Dystonia. Mov Disord Clin Pract. 2015; 2(3): 224-226, doi: 10.1002/mdc3.12172, indexed in Pubmed: 30838229.

30. Jost WH, Tatu L, Pandey S, et al. Frequency of different subtypes of cervical dystonia: a prospective multicenter study according to Col-Cap concept. J Neural Transm (Vienna). 2020; 127(1): 45-50, doi: 10.1007/s00702-019-02116-7, indexed in Pubmed: 31828512.

31. Tatu L, Jost WH. Anatomy and cervical dystonia :. J Neural Transm (Vienna). 2017; 124(2): 237-243, doi: 10.1007/s00702-016-1621-7, indexed in Pubmed: 27624726.

32. Jost WH. Torticaput versus Torticollis: Clinical Effects with Modified Classification and Muscle Selection. Tremor Other Hyperkinet Mov (N Y). 2019; 9, doi: 10.7916/tohm.v0.647, indexed in Pubmed: 31413892.

33. Comella CL, Buchman AS, Tanner CM, et al. Botulinum toxin injection for spasmodic torticollis: increased magnitude of benefit with electromyographic assistance. Neurology. 1992; 42(4): 878-882, doi: 10.1212/wnl.42.4.878, indexed in Pubmed: 1565246.

34. Wu C, Xue F, Chang W, et al. Botulinum toxin type A with or without needle electromyographic guidance in patients with cervical dystonia. Springerplus. 2016; 5(1): 1292, doi: 10.1186/s40064-016-2967-x, indexed in Pubmed: 27547666.

35. Dubinsky RM, Gray CS, Vetere-Overfield B, et al. Electromyographic guidance of botulinum toxin treatment in cervical dystonia. Clin Neuropharmacol. 1991; 14(3): 262-267, doi: 10.1097/00002826199106000-00011, indexed in Pubmed: 2070367.

36. Erdal J, Ostergaard L, Fuglsang-Frederiksen A, et al. Quantitative EMG in botulinum toxin treatment of cervical dystonia. A double-blind, placebo-controlled study. Electroencephalogr Clin Neurophysiol. 1994; 93(6): 434-439, doi: 10.1016/0168-5597(94)90150-3, indexed in Pubmed: 7529693.

37. Østergaard L, Fuglsang-Frederiksen A, Werdelin L, et al. Quantitative EMG in botulinum toxin treatment of cervical dystonia. A double-blind, placebo-controlled study. Electroencephalography and Clinical Neurophysiology/Evoked Potentials Section. 1994; 93(6): 434-439, doi: 10.1016/0168-5597(94)90150-3.

38. Speelman JD, Brans JW. Cervical dystonia and botulinum treatment: is electromyographic guidance necessary? Mov Disord. 1995; 10(6): 802, doi: 10.1002/mds.870100619, indexed in Pubmed: 8750005.

39. Gerpen JV, Matsumoto J, Ahlskog J, et al. Utility of an EMG mapping study in treating cervical dystonia. Muscle \& Nerve. 2000; 23(11): 1752-1756, doi: 10.1002/1097-4598(200011)23:11<1752::aid-mus12>3.0.co;2-u.

40. Werdelin L, Dalager T, Fuglsang-Frederiksen A, et al. The utility of EMG interference pattern analysis in botulinum toxin treatment of torticollis: a randomised, controlled and blinded study. Clin Neurophysiol. 2011; 122(11): 2305-2309, doi: 10.1016/j.clinph.2011.04.012, indexed in Pubmed: 21636319.

41. Nijmeijer SWR, Koelman JH, Standaar TSM, et al. Cervical dystonia: improved treatment response to botulinum toxin after referral to a tertiary centre and the use of polymyography. Parkinsonism Relat Disord. 2013; 19(5): 533-538, doi: 10.1016/j.parkreldis.2013.01.018, indexed in Pubmed: 23466060.

42. Cordivari C, Misra VP, Vincent A, et al. Secondary nonresponsiveness to botulinum toxin $A$ in cervical dystonia: the role of electromyogram-guided injections, botulinum toxin A antibody assay, and the extensor digitorum brevis test. Mov Disord. 2006; 21(10): 1737-1741, doi: 10.1002/mds.21051, indexed in Pubmed: 16874756.

43. Nijmeijer SWR, Koelman JH, Kamphuis DJ, et al. Muscle selection for treatment of cervical dystonia with botulinum toxin--a systematic review. Parkinsonism Relat Disord. 2012; 18(6): 731-736, doi: 10.1016/j.parkreldis.2012.04.005, indexed in Pubmed: 22575237.

44. Bhidayasiri R. Treatment of complex cervical dystonia with botulinum toxin: involvement of deep-cervical muscles may contribute to suboptimal responses. Parkinsonism Relat Disord. 2011; 17 Suppl 1: S20-S24, doi: 10.1016/j.parkreldis.2011.06.015, indexed in Pubmed: 21999891.

45. Fujimoto $H$, Mezaki T, Yokoe M, et al. Sonographic guidance provides a low-risk approach to the longus colli muscle. Mov Disord. 2012; 27(7): 928-9; author reply 929, doi: 10.1002/mds.25024, indexed in Pubmed: 22648450.

46. Sung DH, Choi JY, Kim DH, et al. Localization of dystonic muscles with 18F-FDG PET/CT in idiopathic cervical dystonia. J Nucl Med. 2007; 48(11): 1790-1795, doi: 10.2967/jnumed.107.044024, indexed in Pubmed: 17942812.

47. Lee InHo, Yoon YC, Sung DH, et al. Initial experience with imaging-guided intramuscular botulinum toxin injection in patients with idiopathic cervical dystonia. AJR Am J Roentgenol. 2009; 192(4): $996-$ 1001, doi: 10.2214/AJR.08.1535, indexed in Pubmed: 19304706.

48. Hong JS, Sathe GG, Niyonkuru C, et al. Elimination of dysphagia using ultrasound guidance for botulinum toxin injections in cervical dystonia. Muscle Nerve. 2012; 46(4): 535-539, doi: 10.1002/mus.23409, indexed in Pubmed: 22987694.

49. Schramm A, Bäumer T, Fietzek U, et al. Relevance of sonography for botulinum toxin treatment of cervical dystonia: an expert statement. J Neural Transm (Vienna). 2015; 122(10): 1457-1463, doi: 10.1007/ s00702-014-1356-2, indexed in Pubmed: 25547861.

50. Schramm A, Huber D, Möbius C, et al. Involvement of obliquus capitis inferior muscle in dystonic head tremor. Parkinsonism Relat Disord. 2017; 44: 119-123, doi: 10.1016/j.parkreldis.2017.07.034, indexed in Pubmed: 28802760.

51. Wissel J, Kanovsky P, Ruzicka E, et al. Efficacy and safety of a standardised 500 unit dose of Dysport (clostridium botulinum toxin type A haemaglutinin complex) in a heterogeneous cervical dystonia population: results of a prospective, multicentre, randomised, double-blind, placebo-controlled, parallel group study. J Neurol. 2001; 248(12): 1073-1078, doi: 10.1007/s004150170028, indexed in Pubmed: 12013585.

52. Brans JW, de Boer IP, Aramideh M, et al. Botulinum toxin in cervical dystonia: low dosage with electromyographic guidance. J Neurol. 1995; 242(8): 529-534, doi: 10.1007/bf00867425, indexed in Pubmed: 8530982.

53. Borodic GE, Pearce LB, Smith K, et al. Botulinum a toxin for spasmodic torticollis: multiple vs single injection points per muscle. Head Neck. 1992; 14(1): 33-37, doi: 10.1002/hed.2880140108, indexed in Pubmed: 1624292.

54. Novak I, Campbell L, Boyce M, et al. Cerebral Palsy Institute. Botulinum toxin assessment, intervention and aftercare for cervical dystonia and other causes of hypertonia of the neck: international consensus statement. Eur J Neurol. 2010; 17 Suppl 2: 94-108, doi: 10.1111/j.1468 -1331.2010.03130.x, indexed in Pubmed: 20633181.

55. Sitek EJ, Sołtan W, Wieczorek D, et al. Self-awareness of motor dysfunction in patients with Huntington's disease in comparison to Parkinson's disease and cervical dystonia. J Int Neuropsychol Soc. 2011; 17(5): 788-795, doi: 10.1017/S1355617711000725, indexed in Pubmed: 21729402. 
56. Klingelhoefer L, Chaudhuri K, Kamm C, et al. Validation of a self-completed Dystonia Non-Motor Symptoms Questionnaire. Annals of Clinical and Translational Neurology. 2019; 6(10): 2054-2065, doi: 10.1002/acn3.50900.

57. Pekmezovic T, Svetel M, Ivanovic N, et al. Quality of life in patients with focal dystonia. Clin Neurol Neurosurg. 2009; 111(2): 161-164, doi: 10.1016/j.clineuro.2008.09.023, indexed in Pubmed: 18995953.

58. Fabbrini G, Berardelli I, Moretti G, et al. Psychiatric disorders in adult-onset focal dystonia: a case-control study. Mov Disord. 2010; 25(4): 459-465, doi: 10.1002/mds.22983, indexed in Pubmed: 20108377.

59. Berardelli I, Ferrazzano G, Pasquini M, et al. Clinical course of psychiatric disorders in patients with cervical dystonia. Psychiatry Res. 2015; 229(1-2): 583-585, doi: 10.1016/j.psychres.2015.07.076, indexed in Pubmed: 26239770.

60. Stamelou M, Edwards M, Hallett M, et al. Stamelou M, Edwards M, Hallett M, Bathia K, The non-motor syndrome of primary dystonia: clinical and pathophysiological implications, Brain, 2012 Jun;135(Pt 6):1668-81;doi10. ; 1093: 2015.

61. Hallett M. Neurophysiology of dystonia: The role of inhibition. Neurobiol Dis. 2011; 42(2): 177-184, doi: 10.1016/j.nbd.2010.08.025, indexed in Pubmed: 20817092.

62. Quartarone A, Bagnato S, Rizzo V, et al. Abnormal associative plasticity of the human motor cortex in writer's cramp. Brain. 2003; 126(Pt 12): 2586-2596, doi: 10.1093/brain/awg273, indexed in Pubmed: 14506068.

63. Quartarone A, Pisani A. Abnormal plasticity in dystonia: Disruption of synaptic homeostasis. Neurobiol Dis. 2011; 42(2): 162-170, doi: 10.1016/j.nbd.2010.12.011, indexed in Pubmed: 21168494.

64. Kojovic M, Caronni A, Bologna M, et al. Botulinum toxin injections reduce associative plasticity in patients with primary dystonia. Mov Disord. 2011; 26(7): 1282-1289, doi: 10.1002/mds.23681, indexed in Pubmed: 21469207.

65. Zoons E, Booij J, Delnooz CCS, et al. Randomised controlled trial of escitalopram for cervical dystonia with dystonic jerks/tremor. J Neurol Neurosurg Psychiatry. 2018; 89(6): 579-585, doi: 10.1136/jnnp2017-317352, indexed in Pubmed: 29326295.

66. Dobryansky M, Korsh J, Shen AE, et al. Botulinum toxin type A and B primary resistance. Aesthet Surg J. 2015; 35(2): NP28-NP30, doi: 10.1093/asj/sju027, indexed in Pubmed: 25653242.

67. Thompson JA, Glasgow LA, Warpinski JR, et al. Infant botulism: clinical spectrum and epidemiology. Pediatrics. 1980; 66(6): 936-942, indexed in Pubmed: 7005856.

68. Ferreira J, Colosimo C, Bhidayasiri R, et al. Factors influencing secondary non-response to botulinum toxin type A injections in cervical dystonia. Parkinsonism \& Related Disorders. 2015; 21(2): 111-115, doi: 10.1016/j.parkreldis.2014.09.034.

69. Mohammadi B, Buhr N, Bigalke H, et al. A long-term follow-up of botulinum toxin A in cervical dystonia. Neurol Res. 2009; 31(5): 463-466, doi: 10.1179/174313209X405137, indexed in Pubmed: 19278576.

70. Greene P, Fahn S, Diamond B. Development of resistance to botulinum toxin type A in patients with torticollis. Mov Disord. 1994; 9(2): 213-217, doi: 10.1002/mds.870090216, indexed in Pubmed: 8196686.

71. Simpson DM, Blitzer A, Brashear A, et al. Therapeutics and Technology Assessment Subcommittee of the American Academy of Neurology. Assessment: Botulinum neurotoxin for the treatment of movement disorders (an evidence-based review): report of the Therapeutics and Technology Assessment Subcommittee of the American Academy of
Neurology. Neurology. 2008; 70(19): 1699-1706, doi: 10.1212/01. wnl.0000311389.26145.95, indexed in Pubmed: 18458230.

72. Fabbri M, Leodori G, Fernandes RM, et al. Neutralizing Antibody and Botulinum Toxin Therapy: A Systematic Review and Meta-analysis. Neurotox Res. 2016; 29(1): 105-117, doi: 10.1007/s12640-0159565-5, indexed in Pubmed: 26467676.

73. Naumann M, Carruthers A, Carruthers J, et al. Meta-analysis of neutralizing antibody conversion with onabotulinumtoxinA (BOTOX®) across multiple indications. Mov Disord. 2010; 25(13): 2211-2218, doi: 10.1002/mds.23254, indexed in Pubmed: 20737546.

74. Naumann M, Boo LM, Ackerman AH, et al. Immunogenicity of botulinum toxins. J Neural Transm (Vienna). 2013; 120(2): 275-290, doi: 10.1007/s00702-012-0893-9, indexed in Pubmed: 23008029.

75. Hefter H, Rosenthal D, Moll M. High Botulinum Toxin-Neutralizing Antibody Prevalence Under Long-Term Cervical Dystonia Treatment. Mov Disord Clin Pract. 2016; 3(5): 500-506, doi: 10.1002/mdc3.12322, indexed in Pubmed: 30363520.

76. Albrecht $\mathrm{P}$, Jansen A, Lee Jl, et al. High prevalence of neutralizing antibodies after long-term botulinum neurotoxin therapy. Neurology. 2019; 92(1): e48-e54, doi: 10.1212/WNL.0000000000006688, indexed in Pubmed: 30464031.

77. Jankovic J, Vuong KD, Ahsan J. Comparison of efficacy and immunogenicity of original versus current botulinum toxin in cervical dystonia. Neurology. 2003; 60(7): 1186-1188, doi: 10.1212/01. wnl.0000055087.96356.bb, indexed in Pubmed: 12682332.

78. Benecke R. Clinical relevance of botulinum toxin immunogenicity. BioDrugs. 2012; 26(2): e1-e9, doi: 10.2165/11599840-00000000000000, indexed in Pubmed: 22385408.

79. Dressler D, Tacik P, Saberi FA. Botulinum toxin therapy of cervical dystonia: duration of therapeutic effects. J Neural Transm (Vienna). 2015; 122(2): 297-300, doi: 10.1007/s00702-014-1253-8, indexed in Pubmed: 25052566.

80. Lange $\mathrm{O}$, Bigalke $\mathrm{H}$, Dengler $\mathrm{R}$, et al. Neutralizing antibodies and secondary therapy failure after treatment with botulinum toxin type $A$ : much ado about nothing? Clin Neuropharmacol. 2009; 32(4): 213-218, doi: 10.1097/WNF.0b013e3181914d0a, indexed in Pubmed: 19620852.

81. Fabbri M, Leodori G, Fernandes RM, et al. Neutralizing Antibody and Botulinum Toxin Therapy: A Systematic Review and Meta-analysis. Neurotox Res. 2016; 29(1): 105-117, doi: 10.1007/s12640-0159565-5, indexed in Pubmed: 26467676.

82. Ferreira JJ, Colosimo C, Bhidayasiri R, et al. Factors influencing secondary non-response to botulinum toxin type A injections in cervical dystonia. Parkinsonism Relat Disord. 2015; 21(2): 111-115, doi: 10.1016/j.parkreldis.2014.09.034, indexed in Pubmed: 25499753.

83. Dressler D, Gessler F, Tacik P, et al. An enzyme-linked immunosorbent assay for detection of botulinum toxin-antibodies. Mov Disord. 2014; 29(10): 1322-1324, doi: 10.1002/mds.25956, indexed in Pubmed: 25073795.

84. Kessler KR, Benecke R. The EBD test--a clinical test for the detection of antibodies to botulinum toxin type A. Mov Disord. 1997; 12(1): $95-$ 99, doi: 10.1002/mds.870120116, indexed in Pubmed: 8990060.

85. Berman B, Seeberger L, Kumar R. Long-term safety, efficacy, dosing, and development of resistance with botulinum toxin type $B$ in cervical dystonia. Mov Disord. 2005; 20(2): 233-237, doi: 10.1002/ mds.20290, indexed in Pubmed: 15455449.

86. Dressler D, Bigalke H, Benecke R. Botulinum toxin type B in antibody-induced botulinum toxin type A therapy failure. J Neurol. 2003; 250(8): 967-969, doi: 10.1007/s00415-003-1129-6, indexed in Pubmed: 12928917. 
87. Marin C, Martí MJ, Tolosa E, et al. Muscle activity changes in spasmodic torticollis after botulinum toxin treatment. Eur J Neurol. 1995; 1(3): 243-247, doi: 10.1111/j.1468-1331.1995.tb00078.x, indexed in Pubmed: 24283525.

88. Gelb DJ, Yoshimura DM, Olney RK, et al. Change in pattern of muscle activity following botulinum toxin injections for torticollis. Ann Neurol. 1991; 29(4): 370-376, doi: 10.1002/ana.410290407, indexed in Pubmed: 1929208.

89. Kaňovský P, Dufek J, Halačková $H$, et al. Change in the pattern of cervical dystonia might be the cause of benefit loss during botulinum toxin treatment. Eur J Neurol. 1997; 4(1): 79-84, doi: 10.1111/j.14681331.1997.tb00303.x, indexed in Pubmed: 24283826.

90. Quagliato EM, Carelli EF, Viana MA. A prospective, randomized, double-blind study comparing the efficacy and safety of type a botulinum toxins botox and prosigne in the treatment of cervical dystonia. Clin Neuropharmacol. 2010; 33(1): 22-26, doi: 10.1097/ WNF.0b013e3181c46f48, indexed in Pubmed: 19959960.

91. Pappert EJ, Germanson T. Myobloc/Neurobloc European Cervical Dystonia Study Group. Botulinum toxin type B vs. type A in toxinnaïve patients with cervical dystonia: Randomized, double-blind, noninferiority trial. Mov Disord. 2008; 23(4): 510-517, doi: 10.1002/ mds.21724, indexed in Pubmed: 18098274.

92. Comella CL, Jankovic J, Shannon KM, et al. Dystonia Study Group. Comparison of botulinum toxin serotypes $A$ and $B$ for the treat- ment of cervical dystonia. Neurology. 2005; 65(9): 1423-1429, doi: 10.1212/01.wnl.0000183055.81056.5c, indexed in Pubmed: 16275831.

93. Naumann M, Yakovleff A, Durif F, et al. BOTOX Cervical Dystonia Prospective Study Group. A randomized, double-masked, crossover comparison of the efficacy and safety of botulinum toxin type A produced from the original bulk toxin source and current bulk toxin source for the treatment of cervical dystonia. J Neurol. 2002; 249(1): 57-63, doi: 10.1007/pl00007848, indexed in Pubmed: 11954869.

94. Tarsy D. Comparison of clinical rating scales in treatment of cervical dystonia with botulinum toxin. Mov Disord. 1997; 12(1): 100-102, doi: 10.1002/mds.870120117, indexed in Pubmed: 8990061.

95. Comella CL, Stebbins GT, Goetz CG, et al. Teaching tape for the motor section of the Toronto Western Spasmodic Torticollis Scale. Mov Disord. 1997; 12(4): 570-575, doi: 10.1002/mds.870120414, indexed in Pubmed: 9251076.

96. Espay AJ, Trosch R, Suarez G, et al. Minimal clinically important change in the Toronto Western Spasmodic Torticollis Rating Scale. Parkinsonism Relat Disord. 2018; 52: 94-97, doi: 10.1016/j.parkreldis.2018.03.002, indexed in Pubmed: 29530726.

97. Jost WH, Hefter H, Stenner A, et al. Rating scales for cervical dystonia: a critical evaluation of tools for outcome assessment of botulinum toxin therapy. J Neural Transm (Vienna). 2013; 120(3): 487-496, doi: 10.1007/s00702-012-0887-7, indexed in Pubmed: 22899277. 